九州大学学術情報リポジトリ

Kyushu University Institutional Repository

\title{
A GRAPHICAL ASSOCIATION MEASURE FOR THE CROSS CLASSIFICATION
}

Wakimoto, Kazumasa

Department of Mathematics and Statistics, College of Liberal Arts \& Sciences, Okayama University

https://doi.org/10.5109/13388

出版情報: Bulletin of informatics and cybernetics. 22 (3/4), pp.209-214，1987-03. Research Association of Statistical Sciences

バージョン：

権利関係 : 


\title{
A GRAPHICAL ASSOCIATION MEASURE FOR THE CROSS CLASSIFICATIONS
}

\author{
By
}

\section{Kazumasa W АКІмото*}

\begin{abstract}
An association graph is proposed to the $K \times L$ table of the cross classifications and an association measure based on the graph is also proposed to the table. The association graph makes it easy to find the detailed differences between two $K \times L$ tables. Thus graphical merit is illustrated by the example.
\end{abstract}

\section{Introduction}

Let us consider the finite population which contains a finite number $N$ of units and suppose the population completely known in regard to the classifications. Then we have the $K \times L$ table for the closs classifications shown by the table 1 , where

(i) Classification $A$ divides the population into the $K$ classes $A_{1}, A_{2}, \cdots, A_{K}$.

(ii) Classification $B$ divides the population into the $L$ classes $B_{1}, B_{2}, \cdots, B_{L}$.

(iii) The number of the population units that is classified as both $A_{i}$ and $B_{j}$ is $N_{i j}$.

The marginal numbers are denoted by $N_{i}$. and $N_{. j}$, respectively.

The traditional association measures for the table are mainly as follows. (See Reference [1]).

(i) Mean square contingency: $\quad \phi=\sqrt{\frac{\chi^{2}}{N}}$

Table 1.

\begin{tabular}{|c|c|c|c|c|c|}
\hline \multirow{2}{*}{$A$} & \multicolumn{2}{|c|}{$B$} \\
\cline { 2 - 6 } & $B_{1}$ & $B_{2}$ & $\cdots \cdots \cdots \cdots \cdots$ & $B_{L}$ & Total \\
\hline$A_{1}$ & $N_{11}$ & $N_{12}$ & $\cdots \cdots \cdots \cdots \cdots$ & $N_{1 L}$ & $N_{1 .}$ \\
\hline$A_{2}$ & $N_{21}$ & $N_{22}$ & $\cdots \cdots \cdots \cdots \cdots$ & $N_{2 L}$ & $N_{2 .}$ \\
\hline$\vdots$ & $\vdots$ & $\vdots$ & $\vdots$ & $\vdots$ & $\vdots$ \\
$\vdots$ & $\vdots$ & $\vdots$ & $\vdots$ & $\vdots$ & $\vdots$ \\
\hline$A_{K}$ & $N_{K 1}$ & $N_{K 2}$ & $\cdots \cdots \cdots \cdots \cdots$ & $N_{K L}$ & $N_{K}$. \\
\hline Total & $N_{\cdot 1}$ & $N_{\cdot 2}$ & $\cdots \cdots \cdots \cdots \cdots$ & $N_{\cdot L}$ & $N$ \\
\hline
\end{tabular}

* Department of Mathematics and Statistics, College of Liberal Arts \& Sciences, Okayama University, Tsushima 700 , Japan. 
$\begin{array}{ll}\text { (ii) Coefficient of contingency: } & C=\sqrt{\frac{\chi^{2} / N}{1+\chi^{2} / N}} \\ \text { (iii) Tschuprow's coefficient: } & T=\sqrt{\frac{\chi^{2} / N}{(K-1)(L-1)}} \\ \text { (iv) Cramér's coefficient: } & V=\sqrt{\frac{\chi^{2} / N}{\operatorname{Min}(K-1, L-1)}},\end{array}$ where

$$
\chi^{2}=\sum_{i=1}^{K} \sum_{j=1}^{L} \frac{\left(N_{i j}-\frac{N_{i} \cdot N_{. j}}{N}\right)^{2}}{\frac{N_{i} \cdot N_{. j}}{N}}
$$

In this paper, we proposed a new association measure for the table using the linked line chart which is similar one in the previous papers. (See References [2] and [3]). In the section 2, we discuss how to make an assocation graph using the linked line chart and proposed an association measure based on the graph. In the section 3 , we illustrate the comparison between the new measure and the traditional measures, and illustrate some graphical characteristics for the new measure.

\section{Association Graph}

Put

$$
D_{i j}=\frac{\left(N_{i j}-\frac{N_{i} \cdot N_{\cdot j}}{N}\right)^{2}}{\frac{N_{i \cdot} \cdot N_{\cdot j}}{N}}, \quad(i=1,2, \cdots, K, j=1,2, \cdots, L)
$$

Then we draw an association graph which is constracted by the following steps:

Step 1. Rearrange the values $D_{11}, D_{12}, \cdots, D_{1 L}, D_{21}, D_{22}, \cdots, D_{2 L}, \cdots, D_{K 1}, D_{K 2}, \cdots$, $D_{K L}$ of size $K L$ in the order of the magnitudes as follows:

$$
D(1) \leqq D(2) \leqq \cdots \leqq D(K L-1) \leqq D(K L) .
$$

Step 2. Draw the $x$-axis and the $y$-axis intersecting at right angles at the origin $O$. Draw a line $\overrightarrow{O P}_{1}$ of the length $D(K L)$ in the direction of the $x$-axis starting from the origin $O$. Next, draw another line $\bar{P}_{1} P_{2}$ of the length $D(K L-1)$ in the direction of $\pi / 2(K L-1)$ radian from the line $\overline{O P_{1}}$ starting from the point $P_{1}$. Continue in the same manner and draw other lines ${\overline{P_{2} P_{3}}}_{3},{\overline{P_{K L-1} P_{K L}}}_{\cdots,},{\overline{P_{2 K L-1} P_{2 K L}}}_{\text {of the lengths }}$ $D(K L-2), \cdots, D(1), D(1), \cdots, D(K L)$ in the direction of $\pi / 2(K L-1)$ radian from the before line.

Step 3. Construct a polygon with $4 K L$ sides as shown in Fig. 1, where $P_{i}^{\prime}$ is a symmetric point of $P_{i}(i=1,2, \cdots, 2 K L)$ with respect to the segment $\overline{O P}_{2 K L}$.

We call the chart as shown in Fig. 1 which is constructed by the above steps "Association graph".

Note. From (2.1) and (2.2) we have the following equation.

$$
\overline{O P}_{1}+\bar{P}_{1} P_{2}+\cdots+{\overline{P_{K L-1} P_{K L}}}=\sum_{i=1}^{K L} D(i)=\chi^{2}
$$




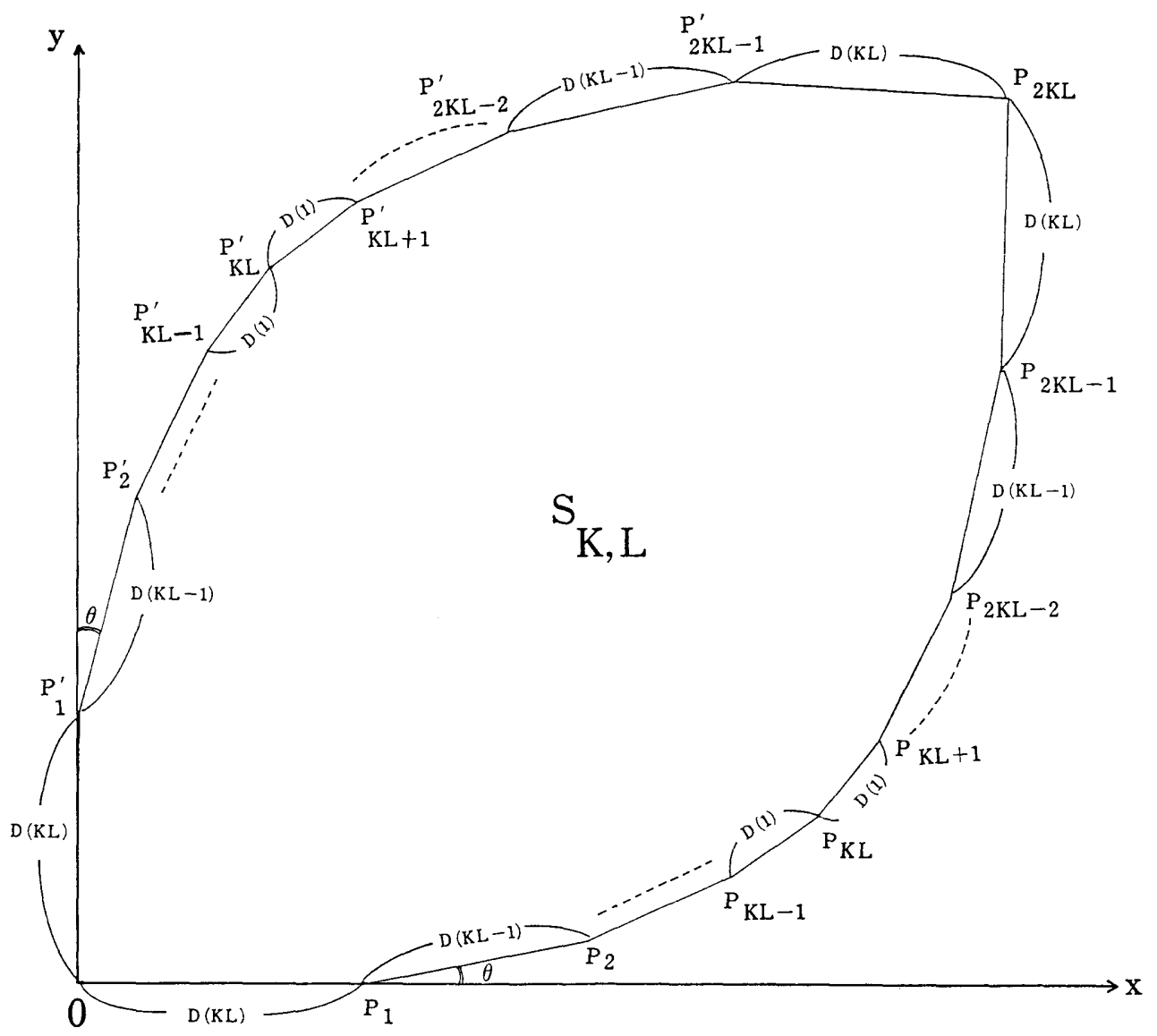

Fig. 1. Association graph, $\theta=\pi / 2(K L-1)$.

\section{An Association Measure Based on the Association Graph}

In Fig. 1, let us denote the area of the polygone which is enclosed by the $4 K L$ sidelines $\overline{O P}_{1},{\overline{P_{1} P_{2}}}_{1} \cdots,{\overline{P_{2 K L-1} P_{2 K L}}}_{\overline{P_{2 K L} P_{2 K L-1}^{\prime}}}, \cdots, \overline{P_{2}^{\prime} P_{1}^{\prime}}, \overline{P_{1}^{\prime} O}$ by $S_{K L}^{2}$. Then we have

$$
\begin{aligned}
S_{K L}^{2}= & 2 \sum_{i=1}^{K L} \sum_{j=1}^{i} D(i) D(j) \sin \frac{i-j}{2(2 K L-1)} \pi \\
& +\sum_{i=1}^{K L} \sum_{j=1}^{K L} D(i) D(j) \sin \frac{i+j-1}{2(2 K L-1)} \pi
\end{aligned}
$$

Put

$$
W_{K L}=\frac{M}{N} S_{K L}
$$

where $M=\max (K, L)$. We call the measure given by (3.2) "Area Coefficient". We disscuss about the upper bound of $W_{K L}$ to lie it between 0 and 1 .

Let us suppose that $K \geqq L$. Let $\Phi$ be the set of all vector $N_{0}=\left(N_{11}, \cdots, N_{L L}, N_{L, L+1}\right.$, $\cdots, N_{L K}$ ) satisfying the following equation:

$$
\sum_{i=1}^{L} N_{i i}+\sum_{i=L+1}^{K} N_{i L}=N .
$$


Then it is well known that the traditional association measures based on the value $\chi^{2}$ are attained equally at any $\boldsymbol{N}_{0}$ in $\Phi$. However, the measure $W_{K L}$ is not equal to all vector $N_{0}$ in $\Phi$. Therefore it may be interesting to find the upper bound. The upper bound of $W_{K L}$ may be attained at a value $N_{0}^{*}$ in $\Phi$. Though we do not obtain the exact upper bound, we have the following inequality in the case of $K=L=E$.

$$
\begin{aligned}
0 \leqq & W_{K L}^{2} \leqq 2\left\{\frac{(E-1)^{4}}{N^{2}} \sum_{i=1}^{E(E-1)} \sum_{j=1}^{i} \sin (i-j) \theta\right. \\
& +\frac{(E-1)^{2}}{N^{2}} \sum_{i=E(E-1)+1}^{E^{2}} \sum_{j=1}^{E(E-1)} \sin (i-j) \theta \\
& \left.+\frac{(E-1)^{4}}{N^{2}} \sum_{i=E(E-1)+1}^{E^{2}} \sum_{j=E(E-1)+1}^{i} \sin (i-j) \theta\right\} \\
& +\frac{1}{N^{2}} \sum_{i=1}^{E(E-1)} \sum_{j=1}^{E(E-1)} \sin (i+j-1) \theta \\
& +\frac{2(E-1)^{2}}{N^{2}} \sum_{i=1}^{E(E-1)} \sum_{j=E(E-1)+1}^{E^{2}} \sin (i+j-1) \theta \\
& +\frac{(E-1)^{4}}{N^{2}} \sum_{i=E(E-1)+1}^{E^{2}} \sum_{j=E(E-1)+1}^{E^{2}} \sin (i+j-1) \theta \\
& +\varepsilon
\end{aligned}
$$

where $\theta=\frac{\pi}{2(M m-1)}$ and the part except $\varepsilon$ in the right hand is the value of $W_{K L}^{2}$.

In the inequality (3.4), $\varepsilon$ may be very small compared with the value of $W_{K L}^{2}$. For example, in the case of $K=L=3$ and $N=9$, we have the Table 2 .

Table 2.

\begin{tabular}{|c|c|c|}
\hline$\left(N_{11}, N_{22}, N_{33}\right)$ & $\chi^{2}$ & $W_{3 \cdot 3}$ \\
\hline$(1,1,7)$ & 18 & 6.271 \\
$(1,2,6)$ & 18 & 6.302 \\
$(1,3,5)$ & 18 & 6.313 \\
$(1,4,4)$ & 18 & 6.312 \\
$(2,2,5)$ & 18 & 6.301 \\
$(2,3,4)$ & 18 & 6.296 \\
$(3,3,3)$ & 18 & 6.251 \\
\hline
\end{tabular}

\section{Example to Show the Graphical Merit}

Assume that two $4 \times 3$ tables are given by the table 3 and table 4 . Then we can draw the association graph as shown by Fig. 2 for the both tables.

In the table 3 and the table 4 , we obtain the association measures including the area coefficient as shown by the table 5 . Then we cannot recognize the difference for the strength of association between the table 3 and the table 4 by looking two measures in the table 5 , because two measures for both tables are very closed. However, we will recognize some difference between both tables by looking two patterns of the 
Table 3 .

\begin{tabular}{|c|c|c|c|c|}
\hline \multirow{2}{*}{$A$} & \multicolumn{4}{|c|}{$B$} \\
\hline & $B_{1}$ & $B_{2}$ & $B_{3}$ & Total \\
\hline$A_{1}$ & 8 & 24 & 35 & 67 \\
\hline$A_{2}$ & 66 & 10 & 18 & 94 \\
\hline$A_{3}$ & 12 & 28 & 41 & 81 \\
\hline$A_{4}$ & 44 & 5 & 9 & 58 \\
\hline Total & 130 & 67 & 103 & 300 \\
\hline
\end{tabular}

Table 4 .

\begin{tabular}{|c|ccc|c|}
\hline$A$ & \multicolumn{4}{|c|}{$B$} \\
\cline { 2 - 5 } & $B_{1}$ & $B_{2}$ & $B_{3}$ & Total \\
\hline$A_{1}$ & 47 & 16 & 4 & 67 \\
\hline$A_{2}$ & 46 & 33 & 15 & 94 \\
\hline$A_{3}$ & 30 & 15 & 36 & 81 \\
\hline$A_{4}$ & 7 & 3 & 48 & 58 \\
\hline Total & 130 & 67 & 103 & 300 \\
\hline
\end{tabular}

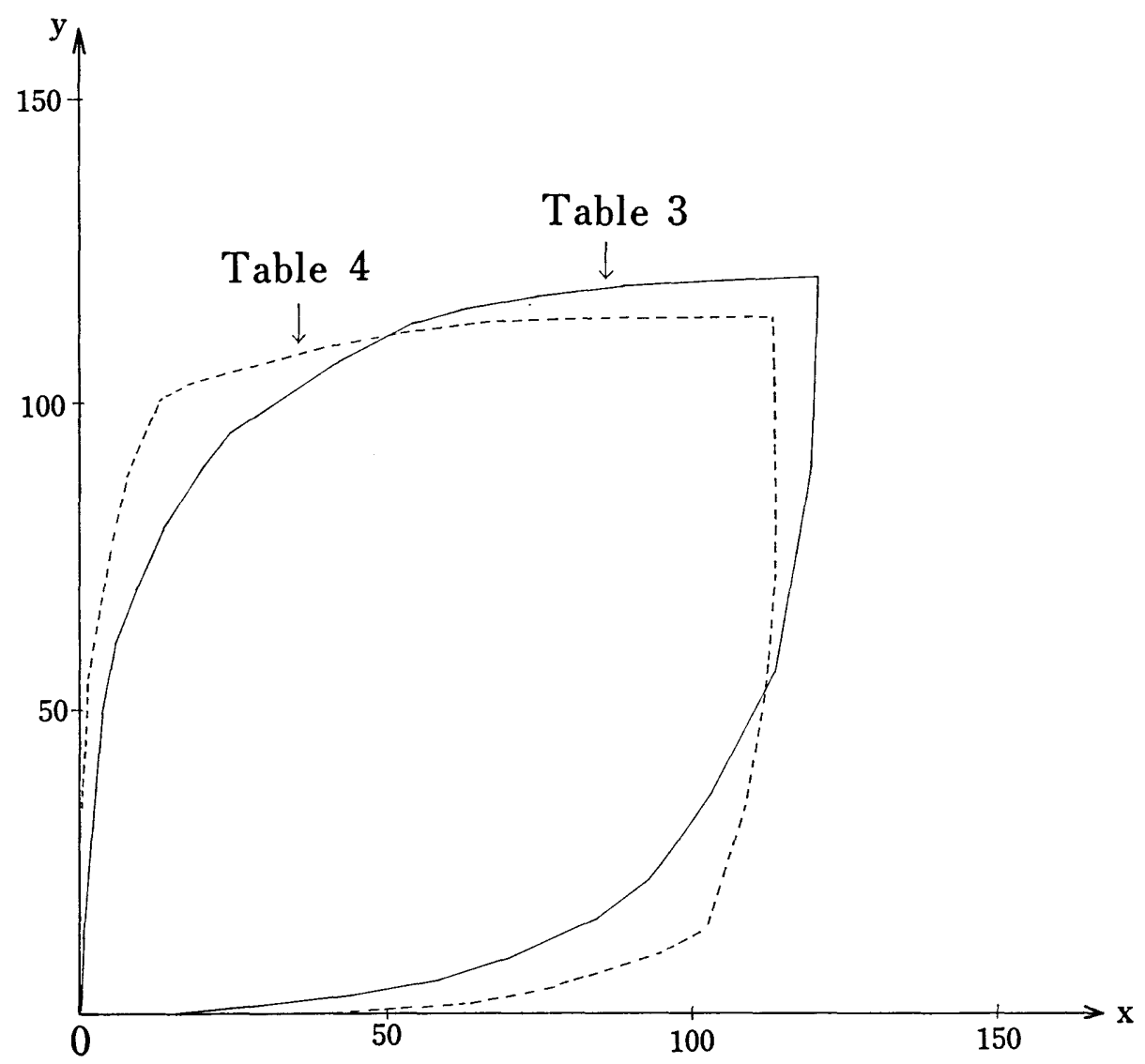

Fig. 2. Association graph for the Table 3 and Table 4.

Table 5. The association measures for the table 3 and the table 4 .

\begin{tabular}{|c|c|c|c|cc|}
\hline & $\phi$ & $C$ & $T$ & $V$ & $W_{3 \times 4}$ \\
\hline Table 3 & 0.594 & 0.511 & 0.243 & 0.420 & 1.46 \\
Table 4 & 0.597 & 0.512 & 0.244 & 0.422 & 1.51 \\
\hline
\end{tabular}


dotted line and the real line in Fig. 2. For example, we will recognize that $D(12)$ and $D(11)$ in the table 4 are larger extremely than these in the table 3.

\section{References}

[1] Goodman, L.A. and Kreskal, W. H.: Measures of Association for Cross Classifications, Springer-Verlag, (1979).

[2] Shirahata, S., Wakimoto, K. and Tarumi, T.: A Goodness of Fit Test Based on the Linked Line Chart, Australian J. Statist., 27 (1985), 163-171.

[3] Wakimoto, K. and Shirahata, S. : A Coefficient of Concordance Based on the Chart of Linked Lines, J. Japan Statist. Soc., 14 (1984), 189-197.

Communicated by Ch. Asano

Received December $2^{\prime 7}, 1985$ 